\title{
Influencia de la adición de carbonato de calcio en la eficiencia energética de la producción de ladrillos de cerámica roja
}

\section{The influence of the addition of calcium carbonate on the energy efficiency of fired clay bricks manufacture}

\author{
Dania Betancourt*1, Fernando M artirena**, Robert Day***, Yosvany Diaz**** \\ * Facultad de Construcciones de la Universidad Central de Las Villas. CUBA \\ ** Subdirector del Centro de Investigación y Desarrollo de las Estructuras y los M ateriales de Construcción (CIDEM) de \\ la Facultad de Construcciones de la U niversidad Central de Las Villas. CUBA \\ *** Universidad de Calgary, Alberta, CANADÁ \\ **** Centro de Investigación y Desarrollo de las Estructuras y los M ateriales de Construcción (CIDEM) de la Facultad de \\ Construcciones de la U niversidad Central de Las Villas. CUBA
}

Fecha de recepción: 20/ 09/2007 Fecha de aceptación: 30/ 11/2007 PAG. $187-196$

Resumen

\begin{abstract}
Se presentan los resultados de un estudio sobre el efecto de la adición de pequeñas cantidades de carbonato de calcio como fundente en la producción de ladrillos rojos de cerámica cocida, con el objetivo de mejorar el perfil energético de la producción a través de la disminución de la temperatura y el tiempo de quema. La adición de cantidades de carbonato de calcio que oscilen entre el 2-5\% de la masa de arcilla mejora la resistencia a compresión de los ladrillos a temperaturas cercanas a los $900^{\circ} \mathrm{C}$, y tiempos de sinterización de 1-3 horas. Este efecto se atribuye a las modificaciones de las reacciones que se producen en las arcillas durante el tratamiento térmico por la presencia del mineral calcita, que permite que la sinterización ocurra a temperaturas más bajas en las arcillas estudiadas, que tienen composición mineralógica donde predomina el mineral motmorillonita. El trabajo hizo un amplio estudio de los cambios en las propiedades físico-químico-mecánicas que se producen a escala microscópica y macroscópica. Estos estudios muestran un incremento consistente de la resistencia mecánica, que se atribuye a la formación de la fase anorthita entre los productos de reacción. Los resultados del trabajo fueron puestos a prueba en talleres prototipo reales, donde se demostró que se producen incrementos de la resistencia a compresión entre 40-50\%, y una reducción de los costos energéticos que oscilan entre un $30-50 \%$.
\end{abstract}

Palabras Clave: Ladrillos cocidos, agentes fundentes, eficiencia energética, propiedades mecánicas, carbonato de calcio

Abstract

The results of a study that assesses the effect of adding small amounts of calcium carbonate as fluxing agent on fired Clay bricks are presented. The paper aims to improve the energy demand of fire clay bricks manufacture through the reduction of firing temperature and process duration. The paper shows that additions in the range of $2-5 \%(\mathrm{wt})$ of clay improves compressive strength of bricks fired at temperature around $900 \stackrel{\circ}{ } \mathrm{C}$ and sintering times between $1-3$ hours. This effect would be caused by modifications of the mineralogical transformations through which clay minerals undergo in the presence of calcite, which reduces sintering temperature in clays containing mainly montmorillonite. This paper presents a broad study of the changes of phisico-chemicalmechanical properties at both macroscale and microscale levels. The results show a consistent increase in compressive strength, associated with the formation of the phase anorthite within the reaction products. These results were tested in real prototypes, where the compressive strength of the manufactured bricks increased by $40-50 \%$, while the energy demand decreased by $30-50 \%$

Keyw ords: Fired Clay bricks, fluxing agents, energy efficiency, mechanical properties, calcium carbonate

\section{Introducción}

Los ladrillos de cerámica roja adquieren la resistencia mecánica a través del tratamiento térmico de la arcilla usada en un proceso conocido como

\footnotetext{
${ }^{1}$ Autor de correspondencia / Corresponding autor:

Departamento de Ing. Civil de la Universidad Central de Las Villas.

Carretera a Camajuaní Km 5 1/2. Santa Clara. Villa Clara. Cuba.

Teléfono: 05342203065, E-mail: daniab@uclv.edu.cu
}

sinterización, el cual se produce cuando los puntos de contacto de los granos adyacentes se funden en una fase vítrea y se unen. Este proceso conocido como sinterización vítrea, comienza a los $800^{\circ} \mathrm{C}$ y continúa hasta temperaturas cercanas a los $1200^{\circ} \mathrm{C}$, cuando tiene lugar la fusión y 
recristalización de los materiales. (Hatzl et al., 2001). Las transformaciones mineralógicas mencionadas anteriormente, se producen sólo a altas temperaturas y con grandes consumos de energía, lo que significa el gasto de considerables volúmenes de combustible (fósiles, leña, biomasa, etc.); se impone por tanto lograr la eficiencia energética en la industria de la cerámica en general y en la producción de ladrillos en particular, bien por la vía del incremento de la eficiencia de los hornos, por la utilización de combustibles más económicos, eficientes, renovables, o por cualquier otra disponible. El incremento de la eficiencia energética puede lograse de manera más económica con el uso de fundentes. Estos son componentes de bajo punto de fusión, que se combinan con los aluminosilicatos presentes en las arcillas y producen cambios en las propiedades mineralógicas de los nuevos productos de reacción, y que colateralmente traen consigo la reducción de la temperatura y el tiempo de cocción, de esta forma contribuyen al ahorro de energía durante la producción. Son conocidas las propiedades fundentes de los carbonatos de calcio y magnesio cuando están presentes en las arcillas. Sin embargo, estas propiedades han sido usadas principalmente en los procesos industriales cuando se desea reducir los cambios dimensionales que se producen en elementos moldeados de arcilla al ser sometidos a altas temperaturas, o cuando se quiere evitar la falla de pilas de ladrillos verdes en el horno durante la quema producto del fenómeno conocido como fallo por fluencia. Es común en estos casos que se busque un compromiso entre la caída de resistencia producida por la adición de carbonato de calcio y el aumento de la carga a la que aparece la fluencia (Elías, 2004).

También se ha reportado en la literatura el uso del carbonato de calcio como aditivo para disminuir el consumo energético en la producción de ladrillos cocidos, esta adición se hace generalmente en gran cantidad, (Cultrone et al., 2000; Jordan et al., 2001) para temperaturas de quema entre 900-1000ㄷ. En estas condiciones hay que tener en cuenta los cambios que introduce la presencia de carbonatos en las propiedades mecánicas de los ladrillos, que consiste básicamente en reducción de resistencia y durabilidad, lo que pone un límite máximo teórico a esta adición, que puede estar normalmente por encima del 10\%, (Elias, 2004).

La acción fundente de los carbonatos (calcita y dolomita) durante el proceso de cocción está asociada a la reducción de la temperatura de sinterización, básicamente porque estos minerales influyen en las transformaciones mineralógicas que tienen lugar en los minerales de arcilla durante su tratamiento térmico y permite la formación de mayor cantidad de fases vítreas a menor temperatura comparado con materiales que carecen de carbonatos, (Cultrone et al., 2000; Mayoral et al., 2001; Jordan et al., 2001). Durante la cocción tienen lugar dos procesos: a) la deshidrolización de la kaolinita $\left(500-600^{\circ} \mathrm{C}\right)$ y b) la descarbonatación de los carbonatos $\left(700-800^{\circ} \mathrm{C}\right)$. U na gran cantidad de material amorfo es creado a través de este proceso, el cual favorece Ia formación de nuevas fases: Anorthita, Ghelenita, Wollastonita (Hatzl et al., 2001; Traore et al.; 2003, Traore y Blanchart, 2003).

Las dosis de carbonatos de calcio y magnesio más usadas en la práctica según la literatura están siempre en el rango 15\%-35\% en relación al peso de la arcilla. En todos estos casos para temperaturas de quema entre 900-1000ㄷ se reportan caídas de la resistencia a compresión producidos por el aumento de la porosidad. Esta porosidad viene de la presencia de grietas por la expansión del óxido de calcio sin combinar cuando se hidrata, y de la misma estructura porosa del óxido de calcio que se forma, (Cultrone et al., 2000; Mayoral et al., 2001; Jordan et al., 2001).

El presente trabajo parte del principio de utilizar pequeñas dosis de carbonato de calcio (menos del 10\% del peso de arcilla) como aditivo fundente en la fabricación de ladrillos cocidos. A este nivel de adición casi todo el carbonato de calcio presente se combina con la arcilla, y la disponibilidad de óxido de calcio sin combinar es menor, por lo que la probabilidad de que se produzca el efecto perjudicial es menor. La presencia del fundente permite bajar la temperatura de quema a valores por debajo de los que normalmente necesita la arcilla en su cocción. De esta forma se puede elevar la eficiencia energética durante el proceso de fabricación, sin el peligro de afectación de las propiedades mecánicas y durabilidad de los ladrillos.

\section{Discusión y desarrollo}

La investigación se desarrolló en dos partes: Primeramente se trabajó en el laboratorio en observar y comprender los cambios mineralógicos y la influencia de esta adición en la durabilidad, en especímenes de arcilla quemados a varias temperaturas en presencia de carbonato de calcio, vistos en la escala microscópica y en la macroscópica. Posteriormente los resultados 
obtenidos en el laboratorio fueron verificados en quemas a escala de pruebas pilotos en hornos reales.

\subsection{O rganización de la experimentación}

Para el estudio se usó una arcilla ordinaria con una composición mineralógica muy heterogénea para el experimento. Esta se obtuvo de una cantera que se encuentra muy cerca de una fábrica de ladrillos ubicada en el municipio de Placetas en la provincia de Villa Clara $y$ ha sido usada por varios años para fabricar ladrillos cocidos. Los ensayos de rayos $X$ a la arcilla indicaron que los minerales presentes en esta son motmorillonita y caolinita (los resultados del ensayo de rayos $X$ a la arcilla usada en el experimento se muestran en la Figura 1). Se adquirió Carbonato de Calcio de alta pureza en el mercado local para ser usado como adición mineral. La tabla 1 muestra la composición química de ambos materiales.

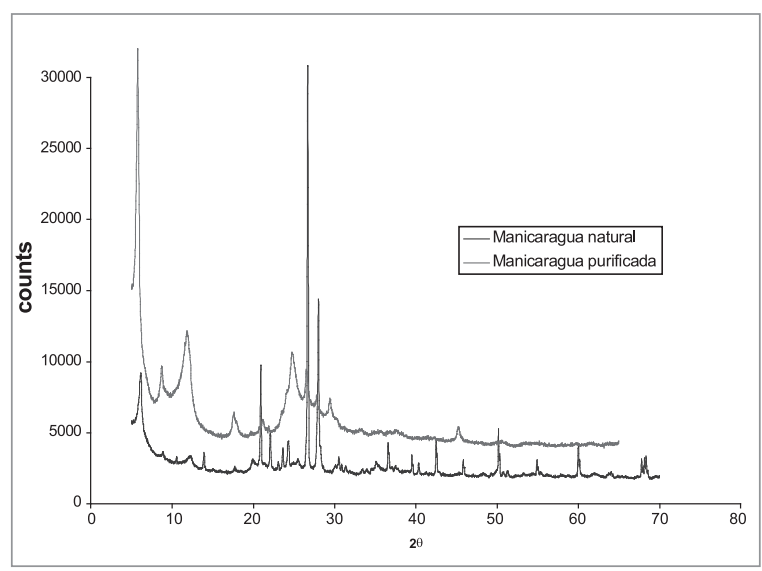

Figura 1. Difractogramas (rayos X) de suelo en su estado natural, y después de un proceso de separación de fracción fina y gruesa

Tabla 1. Composición química de los materiales utilizados en el trabajo a escala de laboratorio

\begin{tabular}{||c|c|c||}
\hline \hline Descripción & Arcilla & $\mathrm{CaCO}_{3}$ \\
\hline $\mathrm{SiO}_{2}$ & 49.56 & 0.99 \\
\hline $\mathrm{Al}_{2} \mathrm{O}_{3}$ & 10.41 & 2.38 \\
\hline $\mathrm{Fe}_{2} \mathrm{O}_{3}$ & 10.73 & 0.90 \\
\hline $\mathrm{TiO}_{2}$ & 0.88 & $<0.02$ \\
\hline $\mathrm{CaO}$ & 3.82 & 52.72 \\
\hline $\mathrm{MgO}$ & 12.32 & 1.89 \\
\hline $\mathrm{Na}_{2} \mathrm{O}$ & 1.39 & $<0.10$ \\
\hline $\mathrm{K}_{2} \mathrm{O}$ & 0.22 & $<0.10$ \\
\hline $\mathrm{P}_{2} \mathrm{O}_{5}$ & 0.07 & 0.05 \\
\hline $\mathrm{MnO}$ & 0.16 & 0.02 \\
\hline $\mathrm{SO}_{3}$ & $<0.10$ & $<0.10$ \\
\hline $\mathrm{LOI}$ & 9.04 & 40.08 \\
\hline
\end{tabular}

La arcilla seca fue mezclada con carbonato de calcio finamente molido hasta una finura de 64 micras (100\% pasado por el Tamiz \#100), en dos proporciones: $2 \%$ y $5 \%$ de la masa total de arcilla. La masa fue humedecida entre un $8-10 \%$, homogeneizada y moldeada en moldes cilíndricos de $3.5 \mathrm{~cm}$ de diámetro, que fueron sometidos a una presión de moldeo de $7 \mathrm{MPa}$. Las probetas cilíndricas del material se secaron a temperatura ambiente por 3-5 días, luego se sometieron a la cocción en un horno eléctrico. De la misma manera se fabricó una serie de control sin CC. La tabla 2 muestra toda la información sobre los especímenes fabricados.

Tabla 2. Muestras ensayadas

\begin{tabular}{||c|c|c|c|c||}
\hline ID & Arcilla $\%$ & CC $\%$ & Temperatura & $\begin{array}{c}\text { Tiempo de } \\
\text { cocción }\end{array}$ \\
\hline M1 & 100 & 0 & $900 \mathrm{C}$ & $2 \mathrm{~h}$ \\
\hline M2 & 98 & 2 & $900 \mathrm{C}$ & $2 \mathrm{~h}$ \\
\hline M3 & 95 & 5 & $900 \mathrm{C}$ & $2 \mathrm{~h}$ \\
\hline M4 & 100 & 0 & $900 \mathrm{C}$ & $3 \mathrm{~h}$ \\
\hline M5 & 98 & 2 & $900 \mathrm{C}$ & $3 \mathrm{~h}$ \\
\hline M6 & 95 & 5 & $900 \mathrm{C}$ & $3 \mathrm{~h}$ \\
\hline M7 & 100 & 2 & $1000 \mathrm{C}$ & $2 \mathrm{~h}$ \\
\hline M8 & 98 & 2 & $1000 \mathrm{C}$ & $2 \mathrm{~h}$ \\
\hline \hline
\end{tabular}

El proceso de cocción se dividió en dos fases: a) La eliminación del agua combinada hasta los $500^{\circ} \mathrm{C}$, y b) Alta temperatura de quema. Los especímenes se mantuvieron por 2 horas en la fase a) y 2 horas en la fase b). Se usaron dos temperaturas de cocción: $900^{\circ} \mathrm{C}$ y $1000^{\circ} \mathrm{C}$ en la fase b). La figura 2 muestra los diferentes regímenes de cocción.

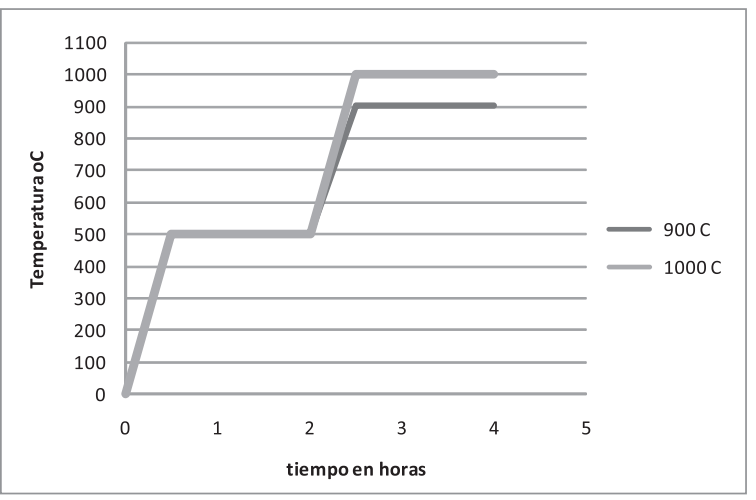

Figura 2. Régimen de cocción de los especímenes fabricados

Los especímenes producidos fueron sometidos a los siguientes ensayos:

1. Resistencia a la compresión, densidad y absorción 
de agua.

2. Medida de la porosidad de los ladrillos a través del ensayo de Porosimetría por Intrusión de Mercurio.

3. Observación de la superficie de la fractura de los ladrillos por el Microscopio Electrónico de Barrido.

4. Análisis mineralógico de muestras finamente molidas de las probetas a través de la difracción por rayos $X$.

5. O bservación de superficies pulidas de los ladrillos en el Microscopio Electrónico Petrográfico y de Barrido.

También a escala de laboratorio se llevó a cabo el estudio de la influencia de la adición en pequeñas cantidades de carbonato de calcio en la durabilidad de los ladrillos cocidos, para ello se elaboraron en este caso ladrillos y no probetas, los que se fabricaron en un taller artesanal y se quemaron con la adición del $5 \%$ de CC en un horno a temperaturas de $900^{\circ} \mathrm{C}$ y $1000^{\circ} \mathrm{C}$ por espacio de tres horas. Los ladrillos ya cocidos fueron sometidos a Resistencia a la compresión, Densidad y Absorción, según la norma cubana NC 54 - 224 - 82: Especificaciones de calidad para el ladrillo estándar. Para evaluar la durabilidad, los especímenes fueron sometidos a un proceso de envejecimiento acelerado según el procedimiento que plantea la norma U NE 7136-58, que consiste en: a) ciclos de humedecimientosecado, y b) cristalización de sales.

\subsection{Trabajo experimental a escala de quemas piloto}

Para realizar el estudio del carbonato de calcio y su influencia en las propiedades de los ladrillos cerámicos producidos en hornos reales, se realizaron quemas prototipo en las que se midió la temperatura, tiempo de duración de quema y consumo de combustible. El análisis y comparación de cada una de estas quemas pilotos se realizó teniendo en cuenta los siguientes aspectos:

1) Calidad del producto

2) El impacto ambiental que trae consigo la adición del carbonato de calcio a la arcilla durante la etapa de cocción.

3) Aspectos económicos.

\section{1) Calidad del producto:}

La calidad de los ladrillos producidos en cada una de las quemas llevadas a cabo en los estudios de caso analizados, se comprobó a partir de la realización de los ensayos que establece la norma cubana: NC 359:2005. Ladrillos y bloques de arcilla cocida-M étodos de ensayo. Los ensayos realizados a ladrillos tomados de
Ios hornos en las cuatro quemas fueron lo siguientes: Resistencia a la compresión, absorción, alabeo, determinación de la masa y determinación de las dimensiones.

2) Impacto ambiental que trae consigo la adición de carbonato de calcio a la arcilla durante la etapa de cocción:

Al adicionar carbonato de calcio finamente molido, se logra la reducción del tiempo de cocción, con lo cual se reducen también los efectos negativos que ocasiona esta producción, fundamentalmente en lo que concierne a la emisión de gases de combustión causantes del efecto invernadero, la cual está íntimamente relacionada con la disminución del consumo de combustible que significa la combustión a más bajas temperaturas y el menor tiempo de duración de esta etapa.

3) Aspectos económicos:

U no de los factores que más incrementa el costo del ladrillo cocido es el gran consumo de combustible que requiere el tratamiento térmico de la arcilla. El mayor o menor consumo de energía depende de varios factores, entre ellos del tipo de combustible empleado, de la eficiencia de los hornos, de la duración de la cocción, entre otros.

Para hacer un estimado del impacto económico del uso del aditivo en la producción de ladrillos, se hizo un esquema de los costos de producción, que incluyó todos los aspectos que influyen en el costo del producto, el cálculo se basó fundamentalmente en la disminución de la cantidad de combustible verificado en cada una de las quemas, si se sabe que el consumo de energía es aproximadamente el $60 \%$ del costo del ladrillo. El esquema de costo empleado se muestra a continuación en la Tabla 3.

Tabla 3. Esquema de costos para la quema sin aditivo

\begin{tabular}{||l|c|}
\hline Componente de costo & Producc. sin aditivo \% \\
\hline Mano de obra & 10 \\
\hline Materias primas & 15 \\
\hline Combustible & 60 \\
\hline Costos indirectos & 15 \\
\hline Total. & 100 \\
\hline
\end{tabular}


Se realizaron en total cuatro quemas piloto, tres de ellas en hornos artesanales del tipo discontinuo 0 intermitente, en fábricas donde la elaboración de los ladrillos es totalmente artesanal, con capacidad de los hornos entre 5000 y 15000 ladrillos.

La última prueba se hizo en una industria clasificada con alta tecnología de producción. Los ladrillos en este caso se cocieron en un horno túnel con capacidad de 120 toneladas a una temperatura controlada de $973^{\circ} \mathrm{C}$ por espacio de 5 horas.

En todas las quemas los ladrillos se produjeron con el 2 y $5 \%$ de adición de carbonato de calcio.

\section{Discusión de los resultados a escala de laboratorio}

\subsection{Propiedades mecánicas}

La figura 3 muestra los valores promedio de resistencia obtenidos en los cilindros ensayados y la desviación estándar correspondiente. Se ensayaron series de 6 especímenes, en los que se produjo una desviación estándar que oscilo entre 2-3 M Pa. Para los cilindros quemados a $900^{\circ} \mathrm{C}$ la adición de pequeñas cantidades de CC fue aparentemente la responsable de un significativo incremento de la resistencia a la compresión, especialmente los hechos con el $2 \%$ de adición. Los resultados cuando se usa un 5\% de CC no son tan altos como los anteriores, aunque son aún superiores a los de las series de control. El incremento del tiempo de cocción de 2 a 3 horas no parece tener influencia en el comportamiento mecánico de las probetas.

Una reacción completamente diferente ocurre en las probetas quemadas $1000^{\circ} \mathrm{C}$. Los valores promedios más altos fueron obtenidos por las series de control sin CC, y la adición de pequeñas cantidades de CC parece decrecer la resistencia a la compresión de las mismas.

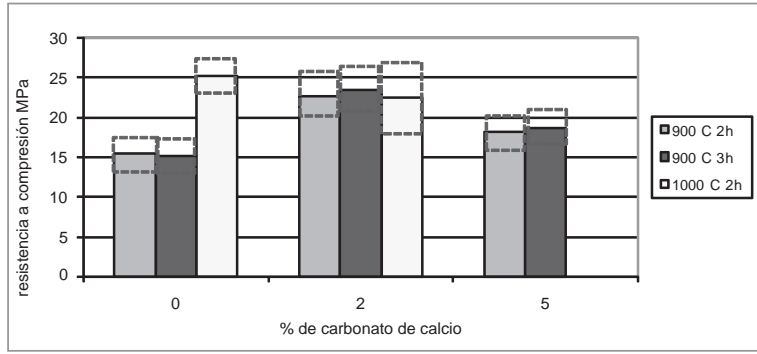

Figura 3. Resultados de los ensayos de Resistencia a la compresión a las probetas y desviación estándar

Contrario a lo que ocurre en las experiencias anteriores referidas (Cultrone et al., 2000; Jordan et al., 2001), donde la adición de carbonato de calcio en proporciones entre el $15-35 \%$ de la masa de arcilla produce caídas de resistencia; la adición de pequeñas dosis de carbonato de calcio permite reducir la temperatura de quema por debajo de la que normalmente necesita la arcilla, sin que se afecten las propiedades mecánicas de los ladrillos. La razón puede estar en que para pequeñas dosis prácticamente todo el carbonato se combina químicamente con la arcilla, y disminuye la cantidad de CaO libre, por lo que las grietas y la porosidad es menor.

Este efecto es más visible a $900 \stackrel{\circ}{ } \mathrm{C}$, temperatura que en la arcilla sin fundente (serie patrón) no ha sido completamente cocida y tiene aún una baja resistencia. La adición de pequeñas dosis de carbonato de calcio permite lograr una sinterización más completa a esta temperatura, lo que se refleja en aumento de la densidad y resistencia de la muestra en relación a la muestra patrón.

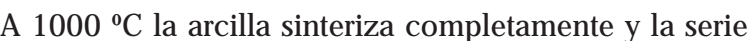
patrón alcanza su resistencia máxima, y la adición de carbonato de calcio produce una ligera caída de resistencia, aparentemente por los cambios en la porosidad que produce la presencia e hidratación del óxido de calcio y el proceso de vitrificación.

\subsection{Estructura de poros}

La Figura 4 muestra los resultados del ensayo de Porosimetría por Intrusión de Mercurio realizado a las probetas cilíndricas con el objetivo de evaluar las características del sistema de poros de los ladrillos. Las series de control quemadas a $900^{\circ} \mathrm{C}$ muestran una distribución de tamaño típica con un pico alrededor de 1 micra (M 1). La cantidad de poros más pequeños así como poros por debajo de una micra se incrementa en muestras con carbonato quemadas a $900^{\circ} \mathrm{C}$ (M 2), aparentemente por dos razones: a) la transformación del Carbonato de calcio en $\mathrm{CaO}$ trae consigo un sistema con poros más pequeños y b) la formación de microfisuras producidas durante la hidratación del CaO (Cultrone at al., 2004).

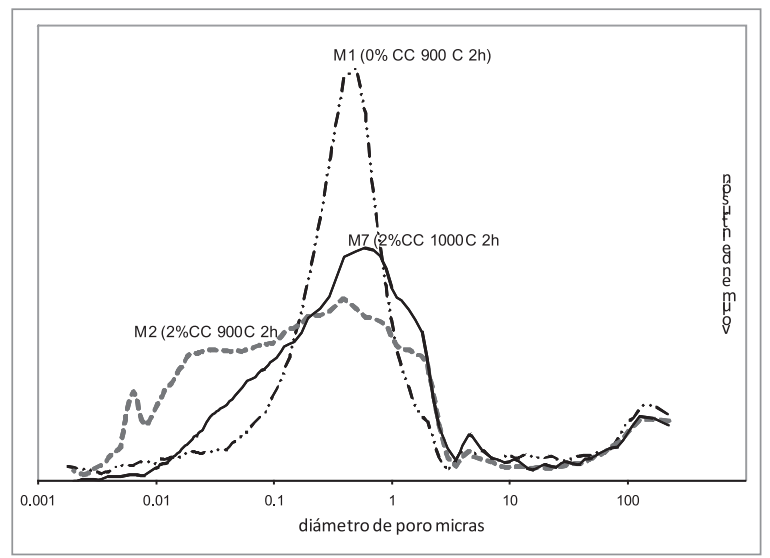

Figura 4. Resultados de los ensayos de MIP de las muestras M 1, M2 y $\mathrm{M} 7$ 
La observación de la superficie de fractura de estos ladrillos en el Microscopio Electrónico, presentada en la Figura 5 , indica claramente la presencia de pequeños poros y también de pequeñas fisuras.

Las probetas hechas con CC y quemadas a $1000^{\circ} \mathrm{C}$, mostraron una típica distribución de radio de poros hacia poros más grandes, quiere decir poros con tamaños por encima de una micra; el incremento del tamaño de los poros está asociado con la formación de material vidriado, que cierra los pequeños poros, y reduce el volumen de sólido, y aumenta el diámetro de los poros (Cultrone et al., 2004). La cantidad de poros más pequeños decrece en comparación con la muestra de $2 \%$ de CC quemada a $900^{\circ} \mathrm{C}$, lo cual podría indicar que la vitrificación a $1000^{\circ} \mathrm{C}$ es mayor que a $900^{\circ} \mathrm{C}$ (Figura. 5) Lo dicho anteriormente es confirmado por la presencia de vidrio en las fotos del Microscopio electrónico de estos ladrillos, presentadas en la Figura $6 \mathrm{a}$, aparentemente producido por la acción fundente del carbonato de calcio. La presencia de los pequeños poros es probablemente causado por las microfisuras producidas durante la hidratación del $\mathrm{CaO}$, como se presenta en la figura $6 \mathrm{~b}$. Las fisuras son más grandes y más frecuentes en comparación con las de los ladrillos quemados $\mathrm{a} 900^{\circ} \mathrm{C}$.

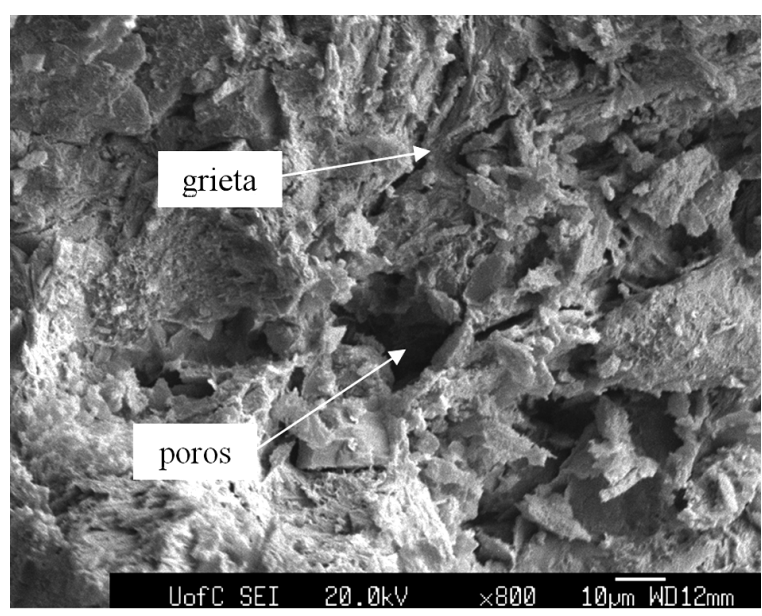

Figura 5. Foto del Microscopio Electrónico a un cilindro hecho con $2 \%$ de Carbonato de calcio y quemado a $900^{\circ} \mathrm{C}$

La Figura 7 muestra los resultados de los ensayos de absorción de agua realizados a las probetas y la desviación estándar correspondiente. Contrariamente a lo que se esperaba, la diferencia entre los resultados de todos los especímenes fue muy pequeña. Los mejores resultados fueron obtenidos por las muestras quemadas a $1000^{\circ} \mathrm{C}$. Este resultado era de esperar a partir del incremento de la vitrificación que se produce, discutida arriba. En todos los casos ni la adición de CC ni el tiempo de cocción parece tener influencia en la absorción de agua. Todos los ladrillos cumplieron los requerimientos de absorción de agua según la norma española UN E 67019 .

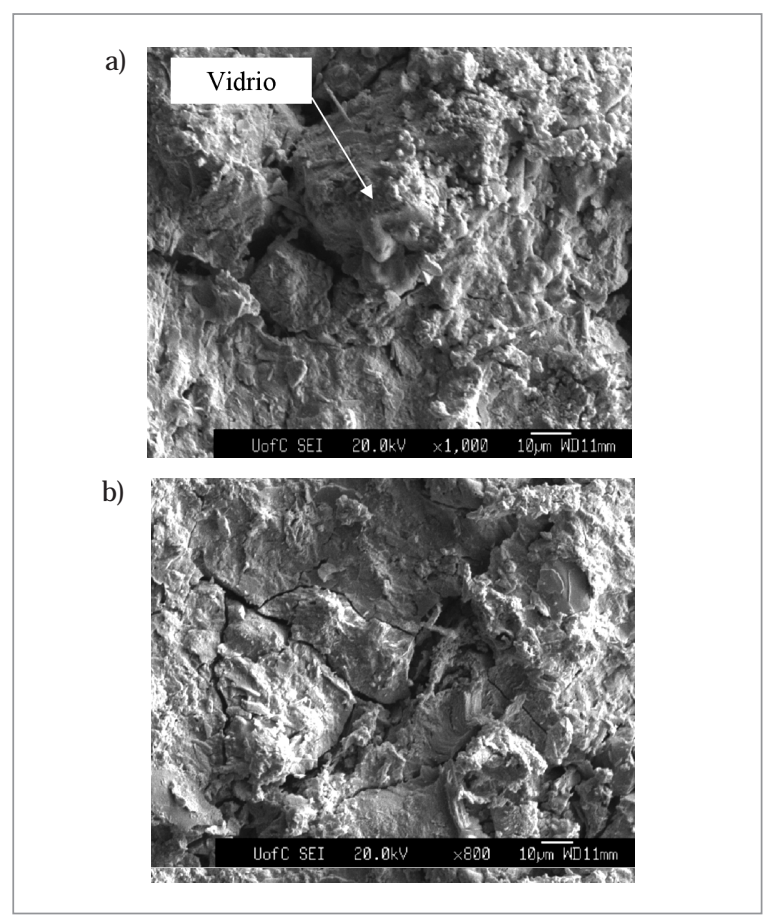

Figura 6. a) Presencia de signos de vidriado en ladrillos hechos con $2 \%$ de CC y quemados a $1000 \stackrel{\circ}{\circ}$, b) Microfisuras en el mismo ladrillo

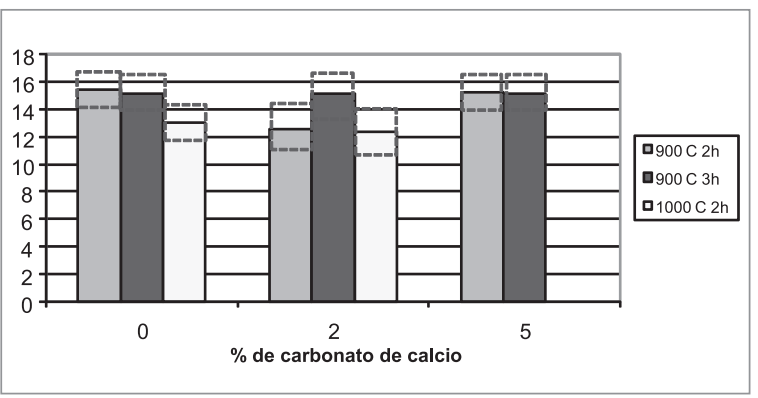

Figura 7. Resultados de los ensayos de Absorción de agua y desviación estándar

\section{Transformaciones mineralógicas}

El mejoramiento de las propiedades mecánicas de los ladrillos y los cambios en la estructura de poros de la matriz resultante puede ser explicado a través de los cambios mineralógicos que tienen lugar cuando se adiciona Carbonato de Calcio, que permiten que el ladrillo alcance su resistencia requerida con una temperatura más baja de combustión, en este caso $900^{\circ} \mathrm{C}$. 


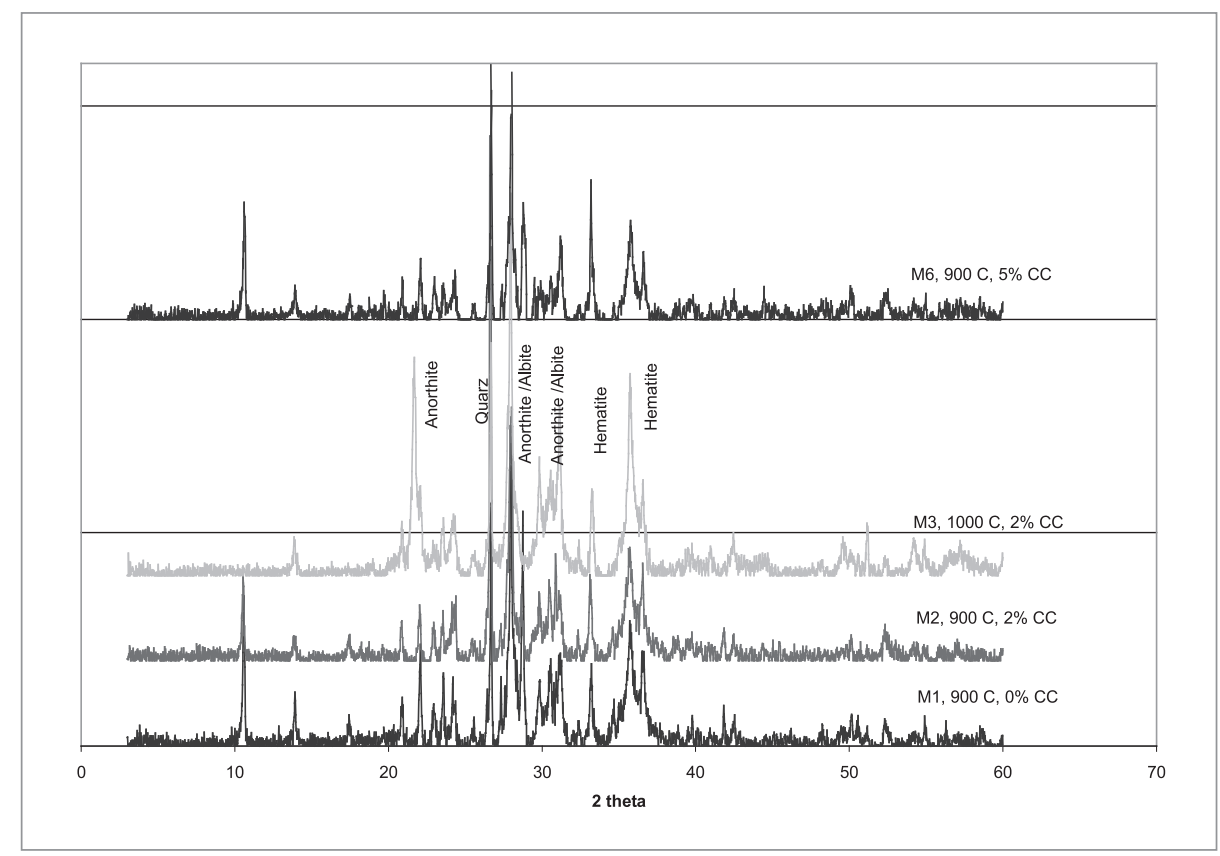

Figura 8. Resultados de los ensayos de Rayos X

Tal como se muestra en la Figura 8, la muestra M 3, quemada a $1000^{\circ} \mathrm{C}$ mostró un nuevo pico a 2-theta 22 grados que concuerda muy bien con la fase Anorthita $\left(\mathrm{CaO} \cdot \mathrm{AL}_{2} \mathrm{O}_{3} \cdot 2 \mathrm{SiO}_{2}\right)$. Este pico no se identifica en la muestra $\mathrm{M} 2$, quemada a $900^{\circ} \mathrm{C}$. Existen también otras bandas anchas en todas las muestras donde la Anorthita - la Albita podrían ser identificadas, probablemente como una fase existente en la arcilla originalmente quemada - como una nueva fase de Anorthita formada. Aunque en el ensayo de difracción de rayos $X$ realizado a todas las muestras no apareció el pico asociado con la fase Anorthita en las muestras quemadas a $900^{\circ} \mathrm{C}$ , aparentemente porque a estas temperaturas esta fase se forma de manera microcristalina. El mapeo de elementos ( $\mathrm{C} a, \mathrm{Al}, \mathrm{Si}$ ) en secciones pulidas, realizado en el JEO L JX8200 Superprobe confirma claramente la presencia de anorthita prácticamente en toda la sección observada (ver figura 9). La anorthita aparece como una red reticular, cuya aparición puede estar relacionada con el aumento de la densidad de la matriz, lo que provoca un aumento relativo de la resistencia en relación a la muestra patrón, en especial a temperaturas más bajas, donde la serie patrón no ha alcanzado aún su temperatura óptima de cocción. A $1000^{\circ} \mathrm{C}$ la resistencia de la serie con carbonato de calcio cae ligeramente en relación a la patrón producto de la vitrificación producida por el carbonato de calcio, que disminuye la densidad y por ende la resistencia, en relación a la serie patrón, que alcanza mayor resistencia por ser cocida a una temperatura más alta. Para el tipo de arcilla analizado, la muestra de menor contenido de carbonato de calcio ( $2 \%$ de la masa de arcilla) brinda los mejores resultados. El incremento del porciento de carbonato de calcio produce una ligera caída de la resistencia, aparentemente porque aumenta la cantidad de material sin combinar, que se convierte a $\mathrm{CaO}$, y es hidratado, se producen mayores grietas de expansión y la densidad de la matriz disminuye.

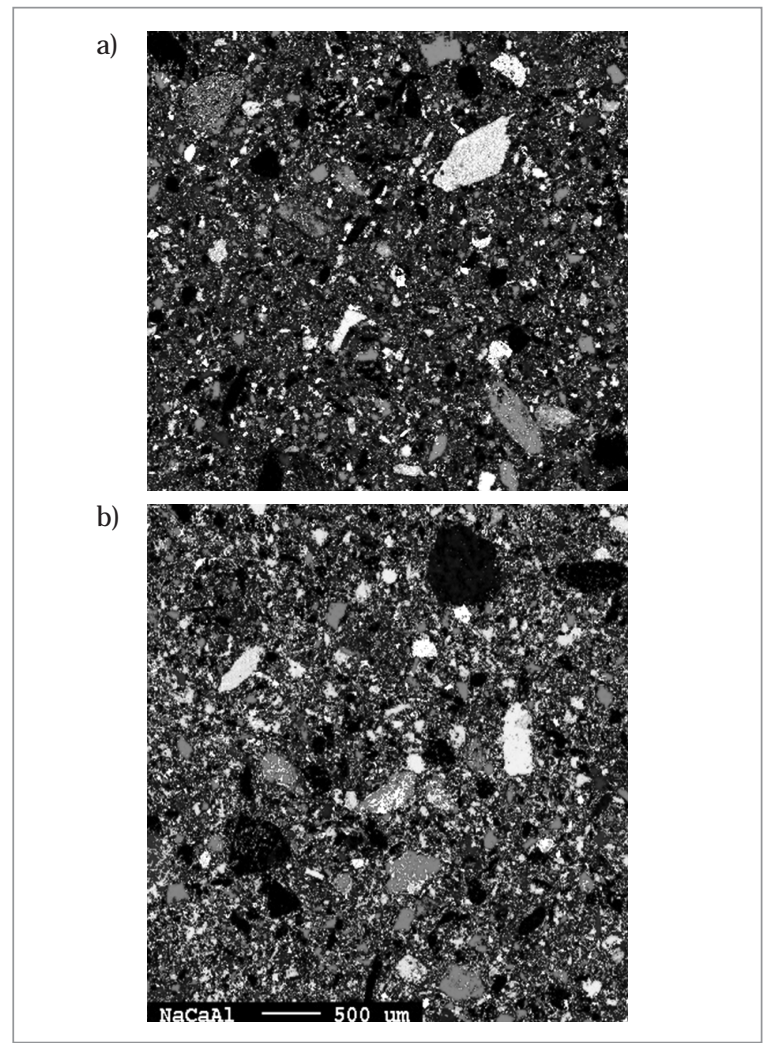

Figura 9. Mapeo de $\mathrm{Na}, \mathrm{Ca}, \mathrm{Al}$. a) muestra $\mathrm{M}-1$, b) muestra $\mathrm{M}-2$ 
4.1 Discusión de los resultados del estudio de durabilidad Los resultados de los ensayos físico-mecánicos realizados a los ladrillos producidos en condiciones reales, fabricados para hacer este estudio, ratificaron los obtenidos por las probetas hechas en el laboratorio. Las muestras fabricadas con adición del $5 \%$ y quemadas a $900^{\circ} \mathrm{C}$ brindan los mejores resultados, y se caracterizan por un aumento de la resistencia a la compresión con respecto a la serie de control (muestra patrón) como se muestra en la Tabla 4. Este aumento es consecuente con el aumento de la densidad que se produce. La absorción se comportó de manera similar en ambas series. Las muestras fabricadas con adición de carbonato y cocidas a $1000^{\circ} \mathrm{C}$ no muestran mejoras de sus propiedades mecánicas, por las razones explicadas arriba.

Tabla 4. Resistencia a Compresión y densidad. Tiempo de Cocción $=3 \mathrm{~h}$

\begin{tabular}{||c|c|c|c|c|c|}
\hline Muestra & $\begin{array}{l}\% \text { de } \\
\mathrm{CaCO}_{3}\end{array}$ & $\%$ de arcilla & $\begin{array}{c}\text { Temp. de } \\
\text { cocción }\end{array}$ & $\begin{array}{c}\text { Res Comp } \\
\mathrm{MPa}\end{array}$ & $\begin{array}{c}\text { Densidad } \\
\left(\mathrm{g} / \mathrm{cm}^{3}\right)\end{array}$ \\
\hline $\mathrm{MP}-1$ & 0 & 100 & $900^{\circ} \mathrm{C}$ & 21.9 & 1.788 \\
\hline $\mathrm{M}-2$ & 5 & 95 & $900^{\circ} \mathrm{C}$ & 33.1 & 1.827 \\
\hline $\mathrm{MP}-3$ & 0 & 100 & $1000^{\circ} \mathrm{C}$ & 23.0 & 1.748 \\
\hline $\mathrm{M}-4$ & 5 & 95 & $1000^{\circ} \mathrm{C}$ & 22.7 & 1.841 \\
\hline
\end{tabular}

4.2 Análisis de resultados del ensayo de los ciclos de humedecimiento y secado

El comportamiento de la muestra patrón y de la muestra con aditivo fue similar ante este ensayo, al no sufrir ninguna de las series pérdidas considerables de peso y en el caso de la inspección visual, no apreciarse fisuras ni grietas.

\subsection{Análisis de resultados del ensayo de Cristalización} de sales

Al analizar los resultados de todas las muestras sometidas a este agresivo ensayo no se detectaron cambios en el comportamiento entre las muestras fabricadas con y sin adición: ambas se deterioraron de forma semejante.

A continuación en la Tabla 5 se muestran los resultados de los ensayos realizados a las series cocidas a 3 horas en el horno eléctrico. Para la experimentación se produjeron series de 6 especímenes, que fueron sometidos a los ensayos descritos arriba.
Tabla 5. Resultados del estudio de durabilidad a ladrillos con sin adición de CC para el tiempo de 3 horas

Ladrillos quemados a $900^{\circ} \mathrm{C}$

\begin{tabular}{|c|c|c|c|c|c|}
\hline Muestras & $\begin{array}{c}\text { Resistencia a } \\
\text { Compresión } \\
(\mathrm{MPa})\end{array}$ & $\begin{array}{c}\text { Absorción } \\
(\%)\end{array}$ & $\begin{array}{c}\text { Densidad } \\
\text { Aparente } \\
\left(\mathrm{g} / \mathrm{cm}^{3}\right)\end{array}$ & $\begin{array}{c}\text { Humedad- } \\
\text { Secado. } \\
\text { Promedio de } \\
\text { Pérdida de peso } \\
\Delta \mathrm{P}(\mathrm{g})\end{array}$ & $\begin{array}{c}\text { Cristal. } \\
\text { de sales } \\
\Delta \mathrm{P}(\mathrm{g})\end{array}$ \\
\hline $\mathrm{MP}-1$ & 21.9 & 13.3 & 1.788 & $\begin{array}{c}\text { Sólo hay } \\
\text { pérdida en una } \\
\text { muestra }=85\end{array}$ & -29 \\
\hline $\mathrm{M}-2$ & 33.1 & 12 & 1.827 & -14.5 & 105.16 \\
\hline \hline
\end{tabular}

Ladrillos quemados a $1000^{\circ} \mathrm{C}$

\begin{tabular}{|c|c|c|c|c|c|}
\hline Muestras & $\begin{array}{c}\text { Resistencia a } \\
\text { Compresión } \\
(\mathrm{MPa})\end{array}$ & $\begin{array}{c}\text { Absorción } \\
(\%)\end{array}$ & $\begin{array}{c}\text { Densidad } \\
\text { Aparente } \\
\left(\mathrm{g} / \mathrm{cm}^{3}\right)\end{array}$ & $\begin{array}{c}\text { Humedad- } \\
\text { secado. } \\
\text { Promedio de } \\
\text { pérdida de peso } \\
\Delta \mathrm{P}(\mathrm{g})\end{array}$ & $\begin{array}{c}\text { Cristal. } \\
\text { de sales } \\
\Delta \mathrm{P}(\mathrm{g})\end{array}$ \\
\hline MP-3 & 23.0 & 13 & 1.748 & -18 & -26.8 \\
\hline $\mathrm{M}-4$ & 22.7 & 13.6 & 1.841 & -24 & -46.16 \\
\hline \hline
\end{tabular}

\section{Discusión de los resultados de las quemas pilotos en hornos artesanales}

1)Calidad del producto:

Los resultados de los ensayos de Resistencia a la compresión a los ladrillos tomados en los hornos artesanales (posterior a la cocción) mostraron un significativo aumento de ésta con respecto a las series de control (sin adición de carbonato de calcio) producidas en esos hornos en similares condiciones de fabricación. Estos aumentos en términos porcentuales están en un rango entre el $15-50 \%$, la absorción se comportó similar en ambas muestras: la patrón y la muestra con adición (2-5\% de CC). La explicación de este resultado puede estar en que los poros adicionales que se produce con el aumento de la dosis de CC no están conectados, y no hacen que aumente la absorción capilar.

2) Impacto ambiental:

De las tres pruebas realizadas en estos hornos, sólo se produjo reducción del tiempo de quema en dos: en una de ellas del $50 \%$ y en la segunda en dos horas y media. La medición de la temperatura en los hornos durante la cocción de los ladrillos reportó temperaturas incluso inferiores a $\operatorname{los} 900^{\circ} \mathrm{C}$, debido a la disminución de la temperatura en los homos y a la reducción del tiempo de quema el consumo de leña (combustible) resultó inferior 
hasta en un $50 \%$, si se compara con quemas realizadas anteriormente a ladrillos sin adición alguna.

\section{3) Aspectos económicos:}

Debido a la disminución del consumo de combustible el costo de producción del ladrillo disminuyó entre el 6 y el 30\% aproximadamente. A continuación en la Tabla 6 se brindan los resultados de las tres quemas pilotos en hornos artesanales.

Tabla 6. Resultados de las quemas artesanales

\begin{tabular}{|c|c|c|c|c|c|}
\hline $\begin{array}{l}\text { Estudio de } \\
\text { caso }\end{array}$ & $\begin{array}{l}\text { Consumo de } \\
\text { combustible }\end{array}$ & $\begin{array}{l}\text { Reducción } \\
\text { del consumo } \\
\text { combustible } \\
\text { con respecto } \\
\text { a la quema } \\
\text { sin aditivo en } \\
\mathrm{Kg} \text { o gls } / \mathrm{h}\end{array}$ & $\begin{array}{l}\text { Tiempo } \\
\text { de } \\
\text { quema }\end{array}$ & Diferencia & $\begin{array}{l}\text { Disminución } \\
\text { del costo total } \\
\text { del ladrillo en } \\
\%\end{array}$ \\
\hline Manicaragua 1 & $\begin{array}{l}3125 \mathrm{~kg}(5000 \\
\text { ladrillos; }(1.6 \\
\text { lad./Kg) }\end{array}$ & $3125 \mathrm{~kg}$. & $\begin{array}{l}5 \text { horas } \\
\text { y media }\end{array}$ & 5 horas & $29.6 \%$ \\
\hline Manicaragua 2 & $\begin{array}{l}2472,75(3700 \mathrm{lad} ; \\
(1.5 \mathrm{lad} / \mathrm{Kg})\end{array}$ & $565.90 \mathrm{~kg}$. & 7 horas & $\begin{array}{l}2 \text { horas } y \\
\text { media. }\end{array}$ & $11.6 \%$ \\
\hline Chambo & $\begin{array}{l}4800 \mathrm{~kg}(15000 \\
\mathrm{lad})(3.1 \mathrm{lad} / \mathrm{Kg})\end{array}$ & $600 \mathrm{~kg}$ & 37 horas & Ninguna. & $6.2 \%$ \\
\hline
\end{tabular}

\section{Resultados de la quema en el horno túnel}

La mayor efectividad del aditivo en cuanto a disminución de la temperatura y consumo de combustible se reportó en el estudio de caso de la fabricación industrial de ladrillos en el horno túnel con temperatura controlada, donde se logró reducir los valores de ésta durante la cocción de $1200^{\circ} \mathrm{C}$ a $973^{\circ} \mathrm{C}$ tal y como se muestra en la Figura 10. Como consecuencia de esta disminución se reportó un ahorro de combustible del $30 \%$ con un incremento en la resistencia a la compresión de las muestras ensayadas del $15 \%$ y una disminución del costo de producción del material del $12 \%$. Los resultados de esta quema piloto se muestran en la Tabla 7.

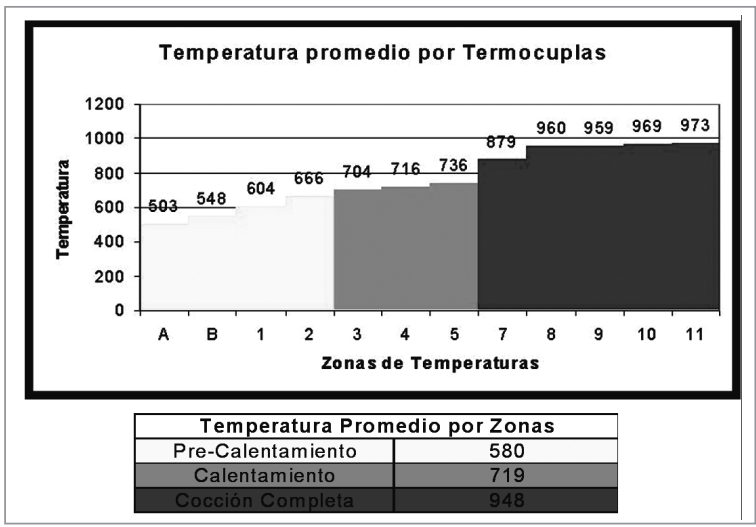

Figura 10. Gráfico de temperatura vs Zonas del horno. (Horno túnel)
Tabla 7. Resultados de la quema piloto realizada en el horno Túnel

\begin{tabular}{|l|l|l|l|l|l||}
\hline $\begin{array}{l}\text { Estudio de } \\
\text { caso }\end{array}$ & $\begin{array}{l}\text { Consumo de } \\
\text { combustible }\end{array}$ & $\begin{array}{l}\text { Reducción } \\
\text { del consumo } \\
\text { combustible } \\
\text { con respecto } \\
\text { a la quema } \\
\text { sin aditivo en } \\
\text { Kg. ogls/h }\end{array}$ & $\begin{array}{l}\text { Tiempo } \\
\text { de } \\
\text { quema }\end{array}$ & Diferencia & $\begin{array}{l}\text { Disminución } \\
\text { del costo total } \\
\text { del ladrillo en } \\
\%\end{array}$ \\
\hline Horno túnel AD & 384 litros/h & 268.8 litros/h & 5 horas & Ninguna. & $12 \%$ \\
\hline
\end{tabular}

\section{7.- Conclusiones}

1. La adición de pequeñas cantidades de carbonato de calcio (por debajo del $5 \%$ ) a la arcilla usada para producir ladrillos cocidos incrementa su resistencia a la compresión en un rango entre el $40-50 \%$. Los mejores resultados se obtuvieron con la adición del $2 \%$.

2. La adición del CC trae consigo un incremento de poros más pequeños (por debajo de una micra). Estos poros provienen de la formación de $\mathrm{CaO}$, y de las microfisuras producidas por la expansión del $\mathrm{CaO}$ durante su hidratación. Además estas grietas son más intensas en las muestras quemadas a $1000^{\circ} \mathrm{C}$, en éstas la cantidad de los poros pequeños decrece, aparentemente porque la acción fundente del carbonato de calcio causa alguna vitrificación y cierra la mayoría de estos.

3. Los resultados de los ensayos a la microestructura mostraron que la fase Anorthita se forma de manera preferente en todos los elementos, y que está bien distribuida en densas áreas, contribuyendo entonces al mejoramiento de la resistencia mecánica de la matriz. Esto es consistente con la composición química de los componentes mezclados y las condiciones en las que se realizó la quema.

4. La adición de pequeñas cantidades de carbonato de calcio no afecta la durabilidad de los ladrillos de cerámica roja, los especímenes fabricados con adición se comportaron de manera similar a aquellos elaborados sin adición alguna ante los ensayos de envejecimiento acelerado.

5. La utilización de Carbonato de calcio como adición permite en general disminuir el consumo energético de la producción de ladrillos con las arcillas estudiadas entre un 30 y un $50 \%$, a la vez que mejora las propiedades físico-mecánicas del producto. Esto representa una significativa contribución a la sostenibilidad económica y ecológica de la producción de ladrillos. 
6. Las aplicaciones a escala de prueba piloto en instalaciones reales de producción de ladrillos confirman los resultados obtenidos a escala de laboratorio y demuestran que la adición de carbonato de calcio puede ser una fuente de aumento de la eficiencia energética de la producción de este material a la vez que permite un incremento de la capacidad productiva de las instalaciones por la disminución del tiempo de cocción.

\section{Agradecimientos}

Los autores desean dar las gracias a la fundación Alejandro de Humboldt de Alemania y a la Universidad de Calgary por el apoyo financiero dado a este trabajo.

\section{Referencias}

Cultrone G., de la Torre M.J., Sebastián E., Cazalla O., Rodríguez Navarro C. (2000), Behaviour of bricks simples in aggresive environments. Water, Air and Soil Pollution 119: 191-207, 2000.

Cultrone G. et al., (2003), Durability of bricks used in the conservation of historic buildings-influence of composition and microstructure. Journal of Cultural Heritage 4 pág 91-99

Cultrone G., Sebastián E., Elert K., de la Torre M.J., Cazalla O., Rodríguez Navarro C. (2004), Influence of mineralogy and firing temperature on the porosity of bricks. Journal of the European Ceramic Society 24 (2004) 547-564

Cultrone G. et al., (2005), M ineralogical and Physical Behavior of Solid Bricks with Additives. Construction and Building Materials 19 (2005) 39-48.

Day R.L, Huizer A. (1994), Fly-Ash Masonry U nit, Report published at the Department of Civil Engineering, University of Calgary, Canada, February 28, 1994.

Day R.L. et al., (1986), Engineering and M icrostructural studies of W estern-Canadian fly ash bricks. Department of Civil Engineering of the U niversity of Calgary. Materials Research Society, Symposia Proceedings, volume 65, December 1986. 11p

Jordán MM et al., (2001), Aptitudes cerámicas de una arcilla tipo modificada por la adición de un residuo rico en carbonato cálcico. Materiales de Construcción 51 No. 261, 2001, pp 5-19.

Karfa Traore, Tibo Simeon Kabre, Philippe Blanchart, (2003)., Gehlenite and anorthite crystallization from kaolinite and calcite mix. Ceramics International 29 (2003) 377-383.
Karfa Traore, Philippe Blanchart (2003), Structural transformation of a kaolinite and calcite mixture to gehlenite and anorthite. Journal of Material Research, Vol. 18, February 2003

Mayoral M.C., Izquierdo M.T., Andres J.M., y Rubio B. (2001), Aluminosilicates transformations in combustion followed by DSC. Thermochimica Acta 373 (2001) 173-180.

Thomas Hatzl, D-München; Peer-L. Gehlken, D. (2001), Ebergötzen Mineralische Rohstoffe in der Ziegelindustrie - Wichtige Parameter in der täglichen Praxis des Geowissenschaftlers ( $M$ ineral raw materials in the brick industry- important parameters for the geologists) (Part 1 and 2). Journal Ziegelindustrie International ZI - 11 and $12 / 2001$ )

Xavier E, (2004), Generación de residues y valoración. Publicado en la Internet en http:// www.cnpml.org/html/archivos/Ponencias/Ponencias ID 56.pdf. 2004 